\title{
Variable Structure-Model Reference Adaptive Control for Stabilization the Attitude of UAV
}

\author{
Zain Anwar $\mathrm{Ali}^{1}$ and Sujeet Pandey ${ }^{2}$. \\ ${ }^{1}$ Collège of Automation Engineering, Nanjing University of Aeronautics \& \\ Astronautics, Nanjing, Jiangsu, China; \\ ${ }^{2}$ Gyancity Research Lab, Motihari, India. \\ *Correspondence: zainanwar86@hotmail.com; Tel.: +86-1301-693-1051, \\ sujeet@gyancity.com
}

\begin{abstract}
In this article, Variable Structure - Model Reference Adaptive Control (VS-MRAC) algorithm with integrator in the feedback loop is proposed called as (VS-MRACI), for stabilization the attitude of the tri-rotor Unmanned Aerial Vehicle (UAV). The proposed controller uses control input as a reference, such that translation and rotational velocities which are used to derive the model of the UAV. The stability of the proposed scheme is presented using Lyapunov candidate function. The validity of the proposed algorithm for stabilizing the attitude of UAV is simulated on Simulink/Matlab. The effectiveness of the proposed algorithm is compared with MRAC. It shows that the proposed algorithm have fast error convergence, robustness and a zero steady state error in the presence of model uncertainties.
\end{abstract}

Keywords: Variable structure, model reference adaptive control, the dual controller and $U A V$.

\section{Introduction}

Autonomous control of flying robots commonly called as Unmanned Aerial Vehicle (UAV) is a challenging task. Since, the last decade it attracts many researchers of the world to work on the designing of rotor crafts as well as its control algorithms [1]. It has an obvious advantage over helicopters due to its rapid direction changing ability, Vertical Takeoff and Landing (VTOL) capability and simple construction. It is widely used in different arenas of our daily life like surveillance, rescue, monitoring of traffic, environmental pollution and for the military secret tasks [2].

UAV's are classified in two types with respect to its body frame, rotary wings and fixed wing [3]. As compared to fixed wing UAV's, rotor craft UAV has two main advantages, VTOL and rapid change in the direction of flight. However, the controlling of rotor crafts is quite complex due to Multiple Input Multi Output (MIMO), highly nonlinear and an under-actuated system along with parameter uncertainties in the dynamic model of the aircraft [4]. The rotor crafts have a wide range of categories including dualrotor, tri-rotor, quad-rotor and hex-rotor. In this paper, our main concern is with the trirotor aerial vehicle that has three rotor fixed at the end of each arm. The tri-rotor aerial robot is an under-actuated system along with four control inputs $\left(u_{1}, u_{2}, u_{3}, u_{4}\right)$ to control the altitude, roll, pitch, yaw and nine outputs: the Euler angles $(\varphi, \theta, \psi)$,the translational velocity $(p, q, r)$ and rotational velocity $(u, v, w)$ with the six degree of freedom (6 DOF). Real time control of the aerial vehicle is required to ensure the stability of the UAV [5]. The tri-rotor aerial vehicle has three motors (actuators) that can be used under the effect of torque to achieve roll and pitch motion [6]. 
In reference [7-10] previously many control algorithms were used to control the dynamics of UAV, based on Fuzzy Logic Control (FLC), Proportional Integral Derivative (PID) control, the Linear Quadratic Regulator (LQR) control. In [11] a nonlinear model of the quadrotor aircraft is controlled by using the adaptive controller which has an ability to give better performance to control the dynamics of the aircraft in the presence of saturation and uncertainties in the model of UAV. In [12-14] the MRAC control algorithm is used to control the model of the quadrotor, in which saturation in the actuator was considered to amplify the linear controller and the stability of the controller was guaranteed by Lyapunov stability criteria.

In [15] a dual controller approach for controlling the dynamics of UAV in which feedback/ feedforward and neural network classifier is designed. A novel scheme is proposed for searching landmark and detection for the autonomous navigation of UAV, such that the key contribution in that article is to combine the entropy of an image with a dual feed-forward / feedback controller for the searching and detection of an object [16]. In reference [17] a nonlinear base dual controller approach is used to control the formation of flight for multiple UAV's under rigid environment. However, the dual controller approach consists of particle swarm optimization (PSO) with state feedback control for the feasibility and stability of multiple UAV's formation flight.

Formerly, many hybrid and dual control algorithms were used to stabilize the dynamics of UAV. In reference [18] gain scheduling PID (GS-PID) with MRAC is used to control the altitude and attitude of UAV. In [19] a novel fuzzy base hybrid algorithm was designed to control the dynamics for the stabilization of tri-rotor UAV. It consists of Regulation, Pole-placement and Tracking (RST) plus Model Reference Adaptive Control (MRAC) such that the gains of the RST controller are being fine-tuned by the fuzzy logic controller. Another hybrid approach was designed for controlling the attitude and altitude of UAV [20]. In which the hybrid controller consists of fuzzy regulation, pole-placement and tracking (F-RST). To stabilize the nonlinear characteristics of UAV, the adaptive hybrid controller is utilized, such that the tuning of the RST controller is achieved by the gains of the adaptive fuzzy logic controller.

In this article, the robustness of VS-MRAC with feedback integrator is compared with classical MRAC of [21] without feedback integrator. To stabilize the attitude of UAV the main contributions are as follows.

i. VS-MRAC is designed by adding the feedback integrator in the controller in which VS-MRAC handles the uncertainties in the system and integrator removes the external disturbance.

ii. The proposed VS-MRACI controller uses translational and rotational velocities as an input constraints, which shows more closeness with real world application.

iii. The stability of overall design of the controller is proved by using Lyapunov stability criteria.

The rest of the article is structured as follows. Section II, defines the system modeling $\&$ its preliminaries are presented. The complete controller designing is described in section III. Section IV defines the simulation, results and discussion. Lastly, section V, concludes the whole article.

\section{System Modeling \& Its Preliminaries}

The tri-rotor aerial robot has three arms and at the end of each arm the rotor is placed. The forces and aerodynamic components of tri-rotor UAV is defined in Figure 1, such that $(\mathrm{X}, \mathrm{Y}, \mathrm{Z})$ axis of $\mathrm{UAV}$ defines the straight direction from the view, the direction at the right side and direction downward from the center of gravity. 


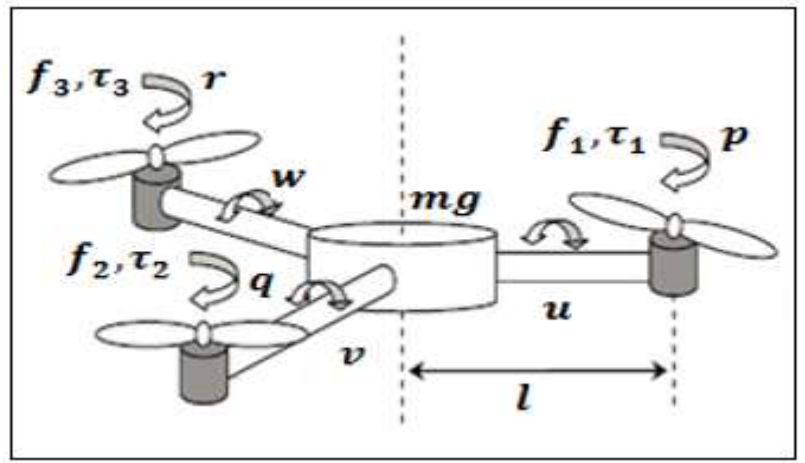

Figure 1. The Structure of the Tri-rotor Aerial Vehicle

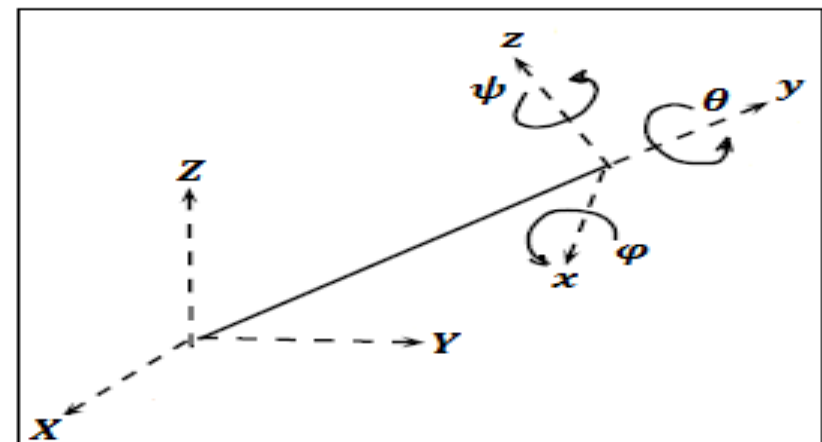

\section{Figure 2. The Relationship between Earth's \& the Tri-Rotor Coordinate System}

The tri-rotor aerial robot coordinate system depends on the Earths coordinate system which defined in Figure 2. The relation between the Earths and the tri-rotor coordinate system are defined by the rotational matrix "R" of the UAV. The roll, pitch and yaw (the attitude of the UAV) or Euler angle which is indicated with $(\varphi, \theta, \psi)$ respectively [22]. The rotation of the UAV is applied with respect to the Earth frame and the complete rotation is done with the roll angle $(\varphi)$ with $\mathrm{X}$ axis, pitch angle $(\theta)$ with $\mathrm{Y}$ axis, and the yaw angle $(\psi)$ with the $\mathrm{Z}$ axis. Now the rotation matrix is defined as,

$$
\left\{\begin{array}{c}
R_{\varphi}^{x} R_{\theta}^{y} R_{\psi}^{z}=\left[\begin{array}{ccc}
1 & 0 & 0 \\
0 & \cos \varphi & \sin \varphi \\
0 & -\sin \varphi & \cos \varphi
\end{array}\right]\left[\begin{array}{ccc}
\cos \theta & 0 & -\sin \theta \\
0 & 1 & 0 \\
\sin \theta & 0 & \cos \theta
\end{array}\right]\left[\begin{array}{ccc}
\cos \psi & \sin \psi & 0 \\
-\sin \psi & \cos \psi & 0 \\
0 & 0 & 1
\end{array}\right] \\
\left.R_{\varphi, \theta, \psi}^{\cos \theta \cos \psi} \quad \begin{array}{ccc}
\cos \theta \sin \psi & -\sin \theta \\
\sin \varphi \sin \theta \cos \psi-\cos \psi \sin \psi & \sin \varphi \sin \theta \sin \psi-\cos \varphi \cos \psi & \sin \varphi \cos \theta \\
\cos \varphi \cos \theta \cos \psi+\sin \varphi \sin \psi & \cos \varphi \sin \theta \sin \psi-\sin \theta \cos \psi & \cos \psi \cos \theta
\end{array}\right]
\end{array}\right.
$$

The actuators, which is fixed in the triangular frame of the aerial robot which produces the thrust force, which helps to hover the UAV. Therefore, the blades of the UAV have fixed rotation angles, such that the direction of the airflow be governed by the rotation of the blades [23]. The overall force and torque of the aerial vehicle is defined as,

$$
\left\{\begin{array}{c}
f_{i}=k t u_{i}^{2} \rightarrow k t u_{i}\left|u_{i}\right| \\
\tau_{i}=k \tau u_{i}^{2} \rightarrow k \tau u_{i}\left|u_{i}\right|
\end{array}\right.
$$

Therefore, $i=1,2,3$ for the rotors of the aerial vehicle.

The rotational or angular velocities and the tilt angle of the aerial robot will offer the UAV to control $U_{S}=\left(u_{1}, u_{2}, u_{3}, u_{4}\right)$ its four movement: such that three are the rotor velocities and the last one is the tilt angle of one rotor out of three. Where as $u_{1}$ to control 
the roll movement, $u_{2}$ to control the pitch movement, $u_{3}$ to control the yaw movement and $u_{4}$ to control the altitude or yaw movement of UAV. To estimate the tri-rotor as a nonholonomic, rigid body, and multivariable along with six Degree Of Freedom (6 DOF), such that the states of the aerial robot is defined as: $(u, v, w),(p, q, r)$ and $(\varphi, \theta, \psi)$ are the linear or translational velocity component, angular or rotational velocity subcomponent and the Euler angles of the body.

The external forces and mass that can be exerted in a body of aircraft which is,

$$
\left\{\begin{array}{c}
F_{\text {ext. }}=\left(\begin{array}{c}
F_{x} \\
F_{y} \\
F_{z}
\end{array}\right)=\left(\begin{array}{c}
0 \\
k t\left(u_{1}\left|u_{1}\right| \operatorname{sinu} u_{4}\right) \\
-k t\left(u_{1}\left|u_{1}\right| \cos u_{4}+u_{2}\left|u_{2}\right|+u_{3}\left|u_{3}\right|\right)
\end{array}\right) \\
(\sqrt{3} / 2) l * k t\left(u_{2}\left|u_{2}\right|-u_{3}\left|u_{3}\right|\right) \\
M_{F_{\text {ext. }}}==\left(\begin{array}{c}
M_{x} \\
M_{y} \\
M_{z}
\end{array}\right)=\left(\begin{array}{c}
0.5 * l * k t\left(u_{2}\left|u_{2}\right|+u_{3}\left|u_{3}\right|\right)-l * k t\left(u_{1}\left|u_{1}\right| \cos u_{4}\right)+k \tau\left(u_{1}\left|u_{1}\right| \operatorname{sinu} u_{4}\right) \\
-l * k t\left(u_{1}\left|u_{1}\right| \operatorname{sinu} u_{4}\right)-k \tau\left(u_{1}\left|u_{1}\right| \operatorname{cosu}_{4}\right)+u_{2}\left|u_{2}\right|+u_{3}\left|u_{3}\right|
\end{array}\right)
\end{array}\right.
$$

Where " $l$ " is the distance from the center of the body to the arm, $\left(u_{1}, u_{2}, u_{3}, u_{4}\right)$ are control inputs of the tri-rotor aerial vehicle. The translational and rotational velocity of the aircraft is described below,

$$
\left\{\begin{array}{c}
\dot{u}=(1 / m)\left(F_{x} \cos \theta \cos \psi+F_{y} \cos \theta \sin \psi-F_{z} \sin \theta\right) \\
\dot{v}=(1 / m)\left(F_{x}(\sin \varphi \sin \theta \cos \psi-\cos \varphi \sin \psi)+F_{y}(\sin \varphi \sin \theta \sin \psi+\cos \theta \cos \psi)+F_{z}(\sin \varphi \cos \theta)\right) \\
\dot{w}=(1 / m)\left(F_{x}(\cos \varphi \sin \theta \cos \psi+\sin \varphi \sin \psi)+F_{y}(\cos \varphi \sin \theta \sin \psi-\sin \varphi \cos \psi)+F_{z}(\cos \varphi \cos \theta)\right)
\end{array}\right.
$$

$$
\left\{\begin{array}{c}
\dot{p}=\left(I_{y}-I_{z} / I_{x}\right)(r * q)+\left(1 / I_{x}\right)(\sqrt{3} * l / 2)\left(f_{2}-f_{3}\right) \\
\dot{q}=\left(I_{z}-I_{x} / I_{y}\right)(r * p)+\left(1 / I_{y}\right)(1 / 2)\left(f_{2}+f_{3}\right)-l * f_{1} \operatorname{cosu}_{4}+\tau_{1} \operatorname{sinu}_{4} \\
\dot{r}=\left(I_{x}-I_{y} / I_{z}\right)(q * p)+\left(1 / I_{z}\right)(1 / 2)\left(f_{2}+f_{3}\right) l * f_{1} \operatorname{sinu} u_{4}-\tau_{1} \operatorname{cosu}_{4}-\tau_{2}-\tau_{3}
\end{array}\right.
$$

Where " $m$ " is the mass, $\left(F_{x}, F_{y}, F_{z}\right)$ the external forces which is exerted on the body $\left(f_{1}, f_{2}, f_{3}\right)$ and $\left(\tau_{1}, \tau_{2}, \tau_{3}\right)$ the forces and torques that is produced by the rotors of the UAV which is already shown in Figure 1.

\section{Controller Designing}

The VS-MRAC was proposed by (Hsu) [24], the main advantages of this algorithm is deal with uncertainties, disturbances and well transient behavior with good robustness. But in this article, VS-MRACI is proposed to control the altitude and attitude of the trirotor aerial robot. The overall working of the control system is shown in Figure 3.

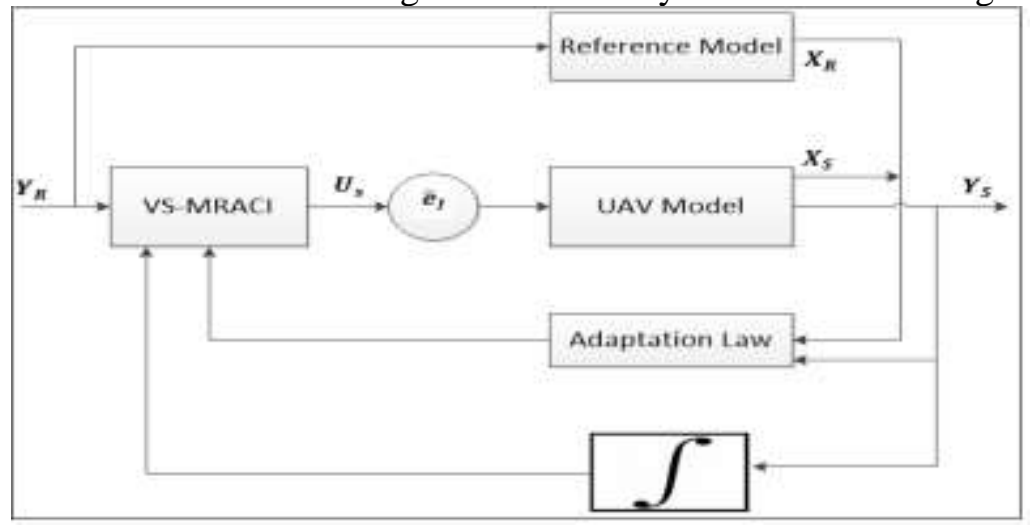

Figure 3. Block Diagram of Control System

Now, the actual model of the system is described as, 


$$
\left\{\begin{array}{c}
\dot{X}_{S}=A_{S} X_{S}+B_{S}+U_{S} \\
Y_{S}=C_{S} X_{S}
\end{array}\right.
$$

The reference model of the system is,

$$
\left\{\begin{array}{c}
\dot{X}_{R}=A_{R} X_{R}+B_{R}+U_{S} \\
Y_{R}=C_{R} X_{R}
\end{array}\right.
$$

The integral base output error is,

$$
\bar{e}_{I}=Y_{S}-Y_{R}
$$

The objective of the controller is to force the actual system output to track the reference output signal, to regulate the $\bar{e}_{I}$ to be zero, although observe the close-loop control signals which are also bounded. Now, the control law which is written as,

$$
U_{s}=\varphi_{1} Y_{s}+\varphi_{2} R
$$

That is same as the classical model reference adaptive control. Furthermore, switching Laws are designed to enhance the system robustness and transient performance of the system. If $B_{S}$ and $A_{S}$ are known, the ideal parameters to control the roll of the UAV are $\left(\bar{\varphi}_{1} \& \bar{\varphi}_{2}\right)$ that can be written as using the following condition.

$$
Y_{S} / R=Y_{R} / R
$$

Equation (10) shows that if our controller fulfills the requirements and the desired path is achieved. Consequently, the controller can be written as,

$U_{s}=\bar{\varphi}_{1} Y_{S}+\bar{\varphi}_{2} R$

To analyze the equation (06) \& (07) the output of the system can be written as.

$$
\left\{\begin{array}{l}
\dot{Y}_{S}=-A_{S} Y_{S}+U_{S} \\
\dot{Y}_{R}=-A_{R} Y_{R}+R
\end{array}\right.
$$

Now using addition and subtraction to make the ideal control parameter of (09).

$$
U_{S}=\varphi_{1} Y_{S}+\varphi_{2} R-\bar{\varphi}_{1} Y_{S}-\bar{\varphi}_{2} R+\bar{\varphi}_{1} Y_{S}+\bar{\varphi}_{2} R
$$

Grouping the terms of the equation (13),

Now,

$$
U_{S}=\left(\varphi_{1}-\bar{\varphi}_{1}\right) Y_{S}+\left(\varphi_{2}-\bar{\varphi}_{2}\right) R+\bar{\varphi}_{1} Y_{S}+\bar{\varphi}_{2} R
$$

$$
U_{S}=\widetilde{\varphi_{1}} Y_{S}+\widetilde{\varphi_{2}} R+\bar{\varphi}_{1} Y_{S}+\bar{\varphi}_{2} R
$$

However, $\widetilde{\varphi_{1}}$ and $\widetilde{\varphi_{2}}$ are the ideal control parameters derivative of $\varphi_{1}$ and $\varphi_{2}$. By substituting (15) in equation (12),

$$
\left\{\begin{array}{c}
\dot{Y}_{S}=-A_{S} Y_{S}+B_{S}\left(\widetilde{\varphi_{1}} Y_{S}+\widetilde{\varphi_{2}} R+\bar{\varphi}_{1} Y_{S}+\bar{\varphi}_{2} R\right) \\
\dot{Y}_{S}=-A_{S} Y_{S}+B_{S} \widetilde{\varphi_{1}} Y_{S}+B_{S} \widetilde{\varphi_{2}} R+B_{S}\left(\bar{\varphi}_{1} Y_{S}+\bar{\varphi}_{2} R\right)
\end{array}\right.
$$

which gives,

$$
\dot{Y}_{S}=-\left(A_{S}+B_{S} \bar{\varphi}_{1}\right) Y_{S}+B_{S} \bar{\varphi}_{2} R+B_{S}\left(\widetilde{\varphi_{1}} Y_{S}+\widetilde{\varphi_{2}} R\right)
$$

Now, from (17) the reference model input can be written as,

$$
R=\left(U_{S}-\bar{\varphi}_{1} Y_{S} / \bar{\varphi}_{2}\right)
$$

By using equation (16) and (18) in the equation (12), we get.

$$
\dot{Y}_{R}(t)=-A_{R} Y_{S}+B_{R} R+\left(B_{R} / \bar{\varphi}_{2}\right)\left(\bar{\varphi}_{1} Y_{S}+\bar{\varphi}_{2} R\right)
$$

Moreover, by comparing (17) \& (16) using equation (10) the reference controller must be,

$$
\left\{\begin{array}{c}
\bar{\varphi}_{1}=\left(A_{S}-A_{R}\right) / B_{S} \\
\bar{\varphi}_{2}=B_{R} / B_{S}
\end{array}\right.
$$

Above the constraints of the system shows that the output converges at the desired state, therefore $B_{S}$ and $A_{S}$ are known, the criteria for designing the controller is called "Matching conditions". Instead of known conditions our main goal are concerned with 
parameters of the system that are unknown, or with parameters of the system along with uncertainties, which need the adaptive laws for regulating the parameters of the actual system.

Now derivate the integral base output error signal of equation (08)

$$
\dot{\bar{e}}_{I}=\dot{Y}_{S}-\dot{Y}_{R}
$$

Now, equation (10) with equations (12), (20) and (21).

$$
\dot{\bar{e}}_{I}=-A_{R} Y_{S}+B_{R} R+\left(B_{R} / \bar{\varphi}_{2}\right)\left(\bar{\varphi}_{1} Y_{S}+\bar{\varphi}_{2} R\right)-\left(-A_{R} Y_{R}+B_{R} R\right)
$$

Rearranging the equation (22),

Thus,

$$
\dot{\bar{e}}_{I}=-A_{R}\left(Y_{S}-Y_{R}\right)+\left(B_{R} / \bar{\varphi}_{2}\right)\left(\widetilde{\varphi_{1}} Y_{S}+\widetilde{\varphi_{2}} R\right)
$$

$$
\dot{\bar{e}}_{I}=-A_{R} * \bar{e}_{I}+\left(B_{R} / \bar{\varphi}_{2}\right)\left(\widetilde{\varphi_{1}} Y_{S}+\widetilde{\varphi_{2}} R\right)
$$

By, considering the lyapunov candidate function,

$$
\dot{V}_{l}\left(\bar{e}_{I}\right)=(1 / 2) *\left(\bar{e}_{I}\right)^{2}>0
$$

Its derivative is,

$$
\dot{V}_{l}\left(\bar{e}_{I}\right)=\bar{e}_{I} * \dot{\bar{e}}_{I}
$$

Now substitute (24) in to (26)

$$
\dot{V}_{l}\left(\bar{e}_{I}\right)=\left(-A_{R} \bar{e}_{I}\right)\left(B_{R} / \bar{\varphi}_{2}\right)\left(\widetilde{\varphi_{1}} Y_{S}+\widetilde{\varphi_{2}} R\right) \bar{e}_{I}
$$

Now, the switching laws for the roll control strategy is written as,

$$
\left\{\begin{array}{l}
\varphi_{1}=-\hat{\varphi}_{1} \operatorname{sgn}\left(\bar{e}_{I} * Y_{S}\right) \\
\varphi_{2}=-\hat{\varphi}_{2} \operatorname{sgn}\left(\bar{e}_{I} * R\right)
\end{array}\right.
$$

The (sgn) function can be written as,

$\therefore \operatorname{sgn}\left(\bar{e}_{I} * Y_{S}\right)=\left\{\begin{array}{l}-1 \text { if }\left(\bar{e}_{I} * Y_{S}\right)<0 \\ 0 \text { if }\left(\bar{e}_{I} * Y_{S}\right)=0 \\ 1 \text { if }\left(\bar{e}_{I} * Y_{S}\right)>0\end{array}\right.$

$\therefore \operatorname{sgn}\left(\bar{e}_{I} * R\right)=\left\{\begin{array}{l}-1 \text { if }\left(\bar{e}_{I} * R\right)<0 \\ 0 \text { if }\left(\bar{e}_{I} * R\right)=0 \\ 1 \text { if }\left(\bar{e}_{I} * R\right)>0\end{array}\right.$

To obtain the desired altitude and attitudes of UAV can be written as,

$$
\left\{\begin{array}{c}
\dot{V}_{l}\left(\bar{e}_{I}\right)=-A_{R} \bar{e}_{I}^{2}-\left(B_{R} / \bar{\varphi}_{2}\right)\left[\left(\hat{Z}_{1}\left|\bar{e}_{I} Y_{S}\right|+\bar{Z}_{1} \bar{e}_{I} Y_{S}\right)+\left(\hat{Z}_{2}\left|\bar{e}_{I} R\right|+\bar{Z}_{2} \bar{e}_{I} R\right)\right] \\
\dot{V}_{l}\left(\bar{e}_{I}\right)=-A_{R} \bar{e}_{I}^{2}-\left(B_{R} / \bar{\varphi}_{2}\right)\left[\left(\hat{\varphi}_{1}\left|\bar{e}_{I} Y_{S}\right|+\bar{\varphi}_{1} \bar{e}_{I} Y_{S}\right)+\left(\hat{\varphi}_{2}\left|\bar{e}_{I} R\right|+\bar{\varphi}_{2} \bar{e}_{I} R\right)\right] \\
\dot{V}_{l}\left(\bar{e}_{I}\right)=-A_{R} \bar{e}_{I}^{2}-\left(B_{R} / \bar{\theta}_{2}\right)\left[\left(\hat{\theta}_{1}\left|\bar{e}_{I} Y_{S}\right|+\bar{\theta}_{1} \bar{e}_{I} Y_{S}\right)+\left(\hat{\theta}_{2}\left|\bar{e}_{I} R\right|+\bar{\theta}_{2} \bar{e}_{I} R\right)\right] \\
\dot{V}_{l}\left(\bar{e}_{I}\right)=-A_{R} \bar{e}_{I}^{2}-\left(\frac{B_{R}}{\bar{\psi}_{2}}\right)\left[\left(\hat{\psi}_{1}\left|\bar{e}_{I} Y_{S}\right|+\bar{\psi}_{1} \bar{e}_{I} Y_{S}\right)+\left(\hat{\psi}_{2}\left|\bar{e}_{I} R\right|+\bar{\psi}_{2} \bar{e}_{I} R\right)\right]
\end{array}\right.
$$

If the conditions,

$\hat{\varphi}_{1}>\left|\bar{\varphi}_{1}\right| \& \hat{\varphi}_{2}>\left|\bar{\varphi}_{1}\right|$ are satisfied for the roll control can be written as,

$\dot{V}_{l}\left(\bar{e}_{I}\right) \leq-A_{R} * \bar{e}_{I}^{2}<0$

The same conditions is designed for pitch, yaw and altitude control.

Which guarantees that " $\bar{e}_{I}=0$ " (GAS) globally asymptotically stable. Equilibrium point become (30) is a negative definite function.

\section{Simulation Results \& Discussion}

This section of the manuscript, defines the robustness and efficiency of the proposed VS-MRACI controller. Moreover, the validity of the designed VS-MRACI controller is compared with the MRAC controller of [21]. According to the model of UAV, the parameters for the simulation conditions are shown in table. 


\section{Table 1. Constraints of UAS}

\begin{tabular}{ccc}
\hline Parameters & Values & Si Units \\
\hline (Mass)“m” & 0.785 & $\mathrm{~kg}$ \\
(G.F) “g” & 9.81 & $\mathrm{~m} / \mathrm{s}^{2}$ \\
$\mathbf{I}$ & 0.3050 & $\mathrm{~m}$ \\
$\mathbf{I}_{\mathbf{x}}$ & 0.3105 & $\mathrm{Kgm}^{2}$ \\
$\mathbf{I}_{\mathbf{y}}$ & 0.2112 & $\mathrm{Kgm}^{2}$ \\
$\mathbf{I}_{\mathbf{z}}$ & 0.2215 & $\mathrm{Kgm}^{2}$ \\
$\boldsymbol{K}_{\boldsymbol{t}}$ & 0.007 & $\mathrm{Nms} 2 / \mathrm{rad} 2$ \\
$\boldsymbol{K}_{\boldsymbol{\tau}}$ & 0.0156 & $\mathrm{Nms} 2 / \mathrm{rad} 2$ \\
\hline
\end{tabular}

The desired state of the UAV uses the input control commands as a reference signals, which are constant and the location of UAV is stable, such that the translational and rotational velocity components are also used to reach the UAV at desired location. The overall performance of the system is governed by VS-MRACI and the stability is ensured by Lyapunov candidate function. The initial position of the UAV is zero and it will reach at the desired height about $1 \mathrm{~m}$ which is shown in Figure 4.

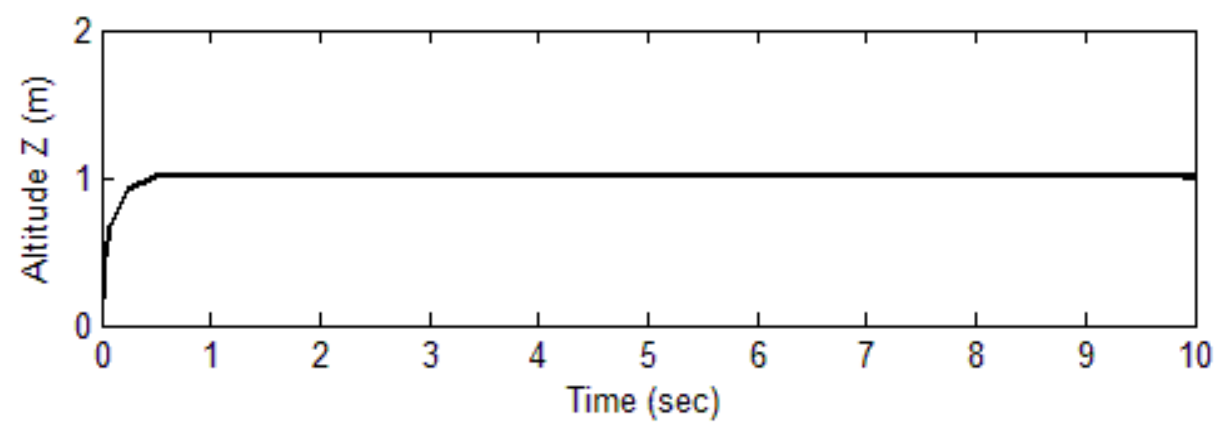

Figure 4. The Desired Altitude of UAV
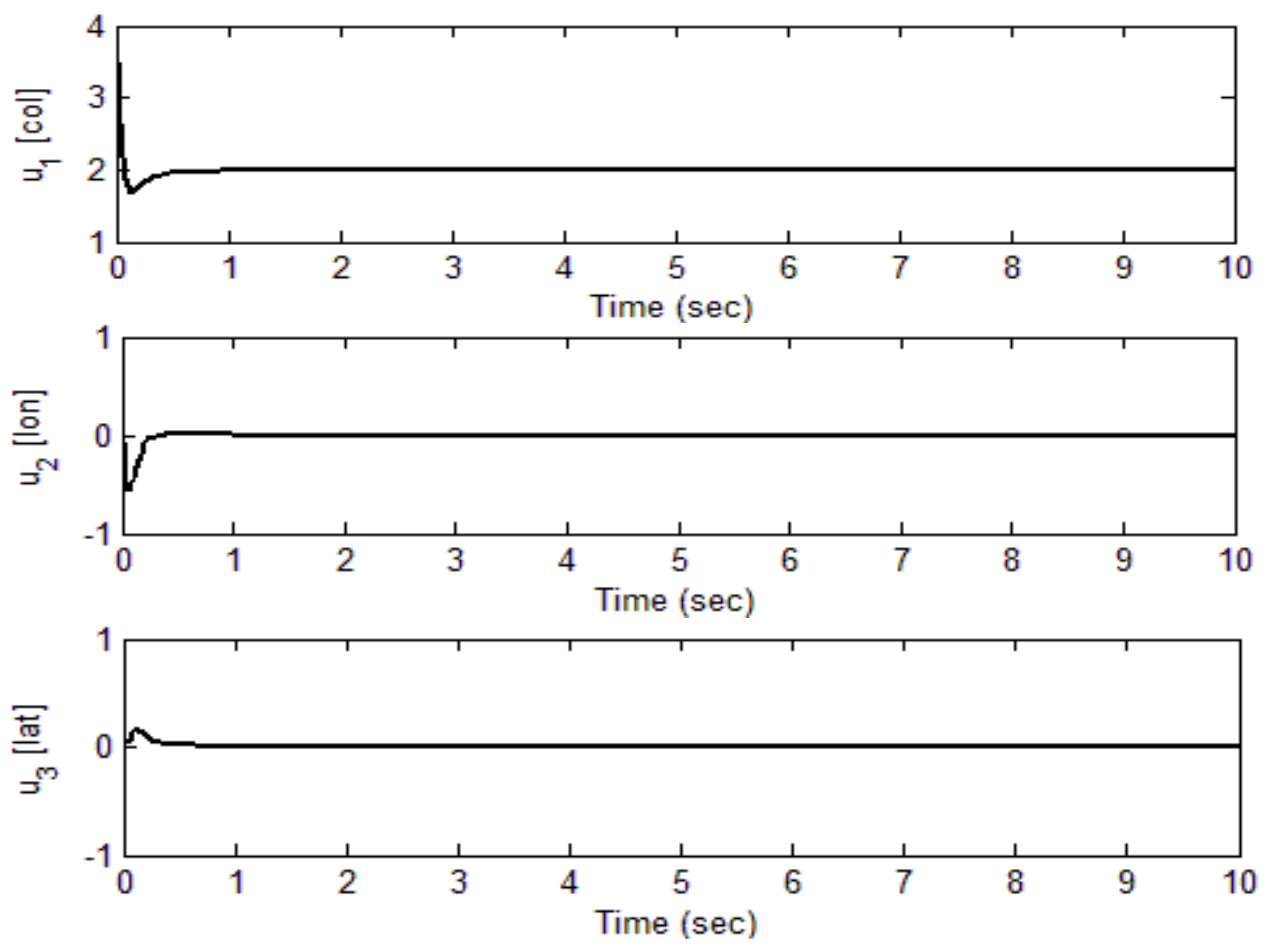


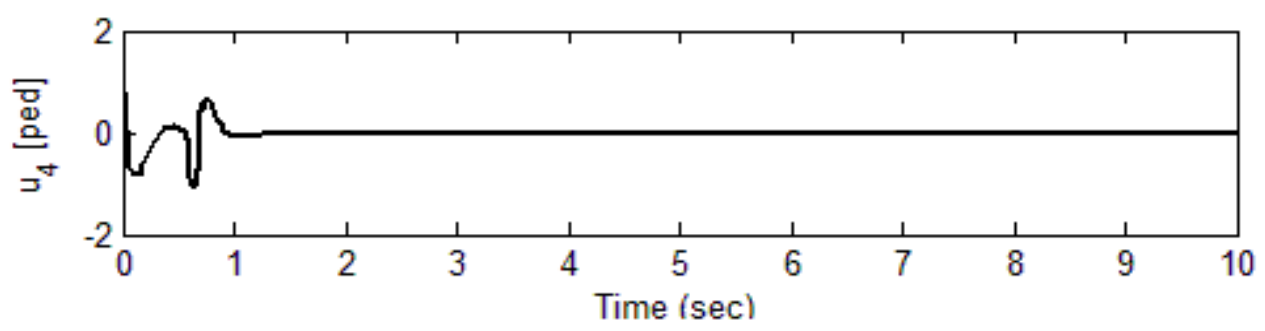

Figure 5. Control Input Commands of UAV

Figure 5, shows the control input ( $\mathrm{u} 1, \mathrm{u} 2, \mathrm{u} 3, \mathrm{u} 4)$ responses of the UAV, initially it has some oscillations and after that it will converge to the desired $(2,0,0$, and 0$)$ input path and behaves linearly. Furthermore, $u_{1} \neq 0$ which confirms that is time invariant in a finite period of time. The initial altitude of the UAV is $(z=0)$ and $(-1,-0.01,-0.01) \mathrm{rad}$, after that the desired position and attitude are: $(\mathrm{z}=1) \mathrm{m}$ and $(\varphi=\theta=\psi=0) \mathrm{rad}$, which is shown in Figure 6. The proposed algorithm stabilize the UAV at predefined altitude and attitude in a fair amount of time. The altitude $\mathrm{z}$ and yaw rate $\psi$ are prominently shows that it converge to the desired states. Moreover, the altitude $\mathrm{z}$, reaches to the predefined value at $\mathrm{u} 1$. The roll and yaw rates have little oscillations to converge at the referred state, which is shown in Figure 6.
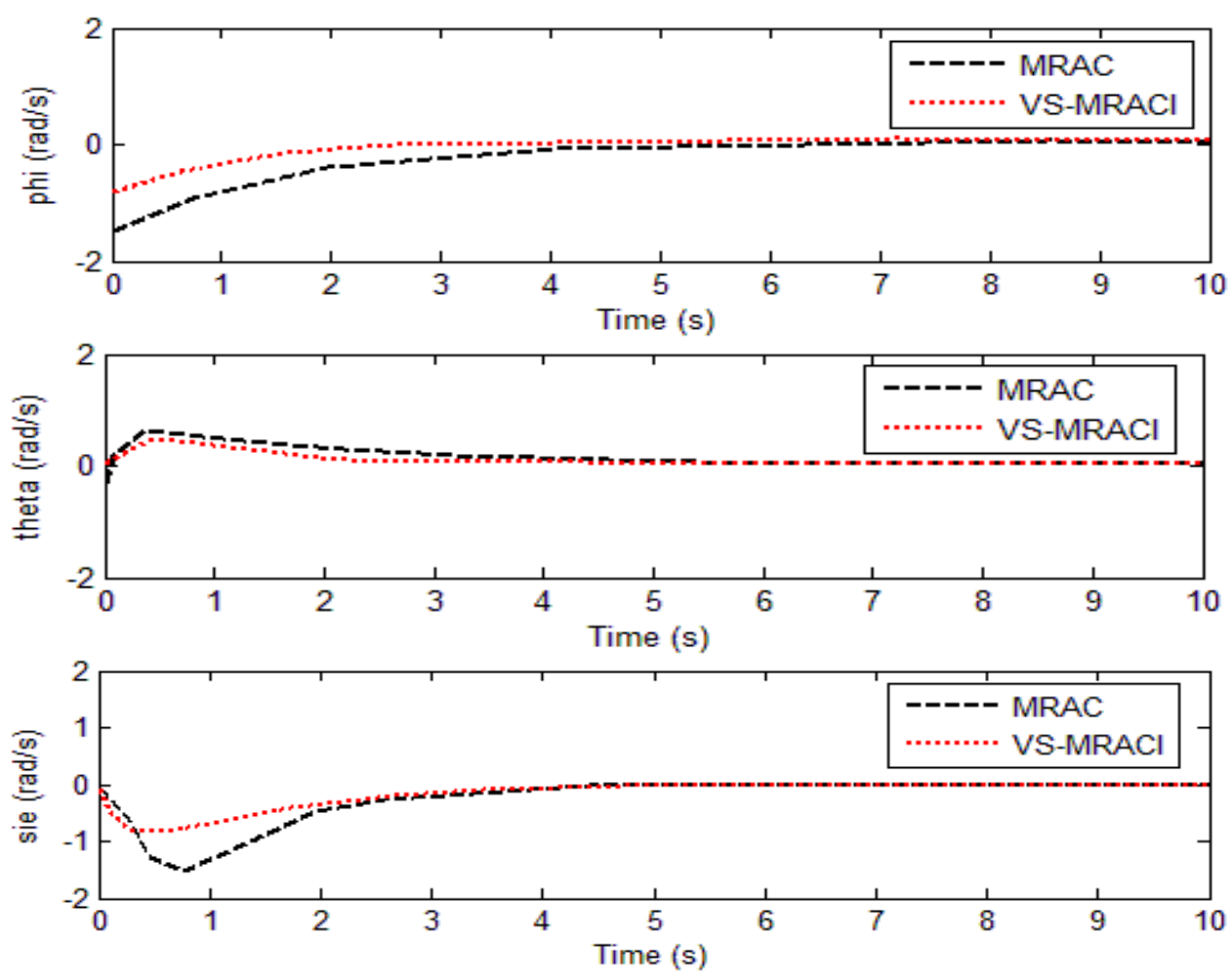

Figure 6. The Euler Angle Rotational Rates of UAV 

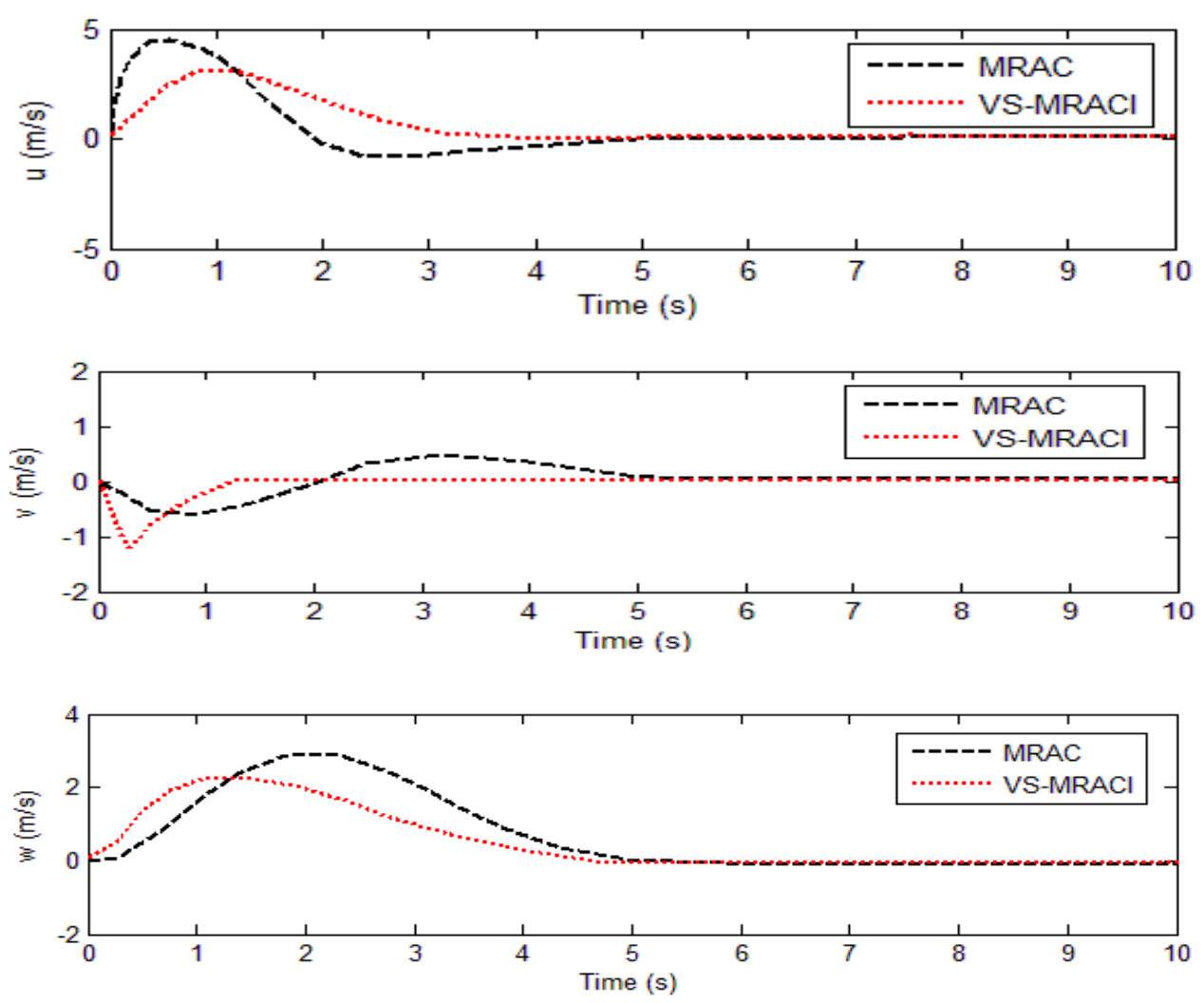

Figure 7. Translational Velocity Components
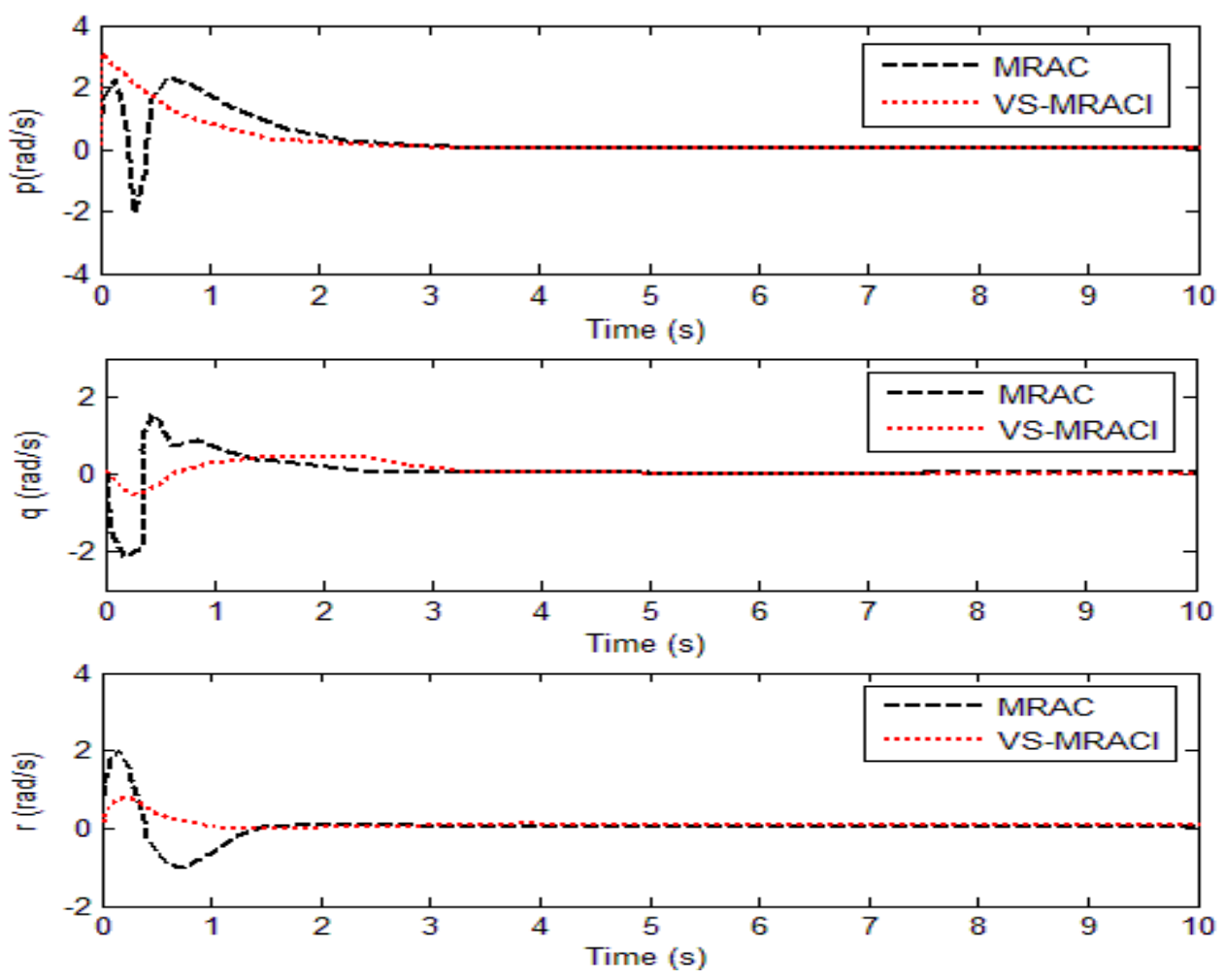

Figure 8. Rotational Velocity Components 
Figure 7 shows the translation velocity components with the initial values of $(u=0.1$ $\mathrm{m} / \mathrm{s}, \mathrm{v}=-0.14 \mathrm{~m} / \mathrm{s}, \mathrm{w}=0.15 \mathrm{~m} / \mathrm{s}$ ) and stabilizes about 5 seconds with little overshoot of "w" velocity component. Figure 8 shows the rotational velocity components with the initial conditions are $(\mathrm{p}=4 \mathrm{rad} / \mathrm{s}, \mathrm{q}=-0.12 \mathrm{rad} / \mathrm{s}, \mathrm{r}=1 \mathrm{rad} / \mathrm{s})$ and will converge to zero in a fair amount of time with any overshoot.

\section{Conclusion}

In this article, a variable structure-model reference adaptive control with integrator in the feedback loop is proposed for the tri-rotor aerial vehicle. Moreover, the modified VSMRACI controller is used to stabilize the attitude of UAV. The proposed algorithm uses translation and rotational velocities to derive the system model. The validity of the controller is illustrated using Simulink/ Matlab and shows that the proposed scheme has good transient behavior with very zero steady state errors to reach at the desired altitude. Additionally, proposed algorithm shows fast convergence and better robustness in the presence of model uncertainties.

\section{Conflicts of Interest}

The authors declare no conflict of interest.

\section{References}

[1] G. Cai, B. M. Chen and T. H. Lee, "An overview on development of miniature unmanned rotorcraft systems", Frontiers of Electrical and Electronic Engineering in China, vol. 5, no. 1, (2010), pp. 1-14.

[2] S. Azeem, "Autonomous Unmanned Aerial Vehicles", Technical report, The George Washington University, (2012).

[3] E. Çetinsoy, S. Dikyar, C. Hançer, K. T. Oner, E. Sirimoglu, M. Unel and M. F. Aksit, "Design and construction of a novel quad tilt-wing UAV", Mechatronics, vol. 22, no. 6, (2012), pp. 723-745.

[4] N. Amiri, "Control of an Unconventional VTOL UAV for Complex Maneuvers", PhD diss., University of Calgary, (2013).

[5] P. Castillo, A. Dzul and R. Lozano, "Real-time stabilization and tracking of a four-rotor mini rotorcraft", IEEE Transactions on control systems technology, vol. 12, no. 4, (2004), pp. 510-516.

[6] S. Bouabdallah, P. Murrieri and R. Siegwart, "Design and control of an indoor micro quadrotor", In Robotics and Automation, 2004. Proceedings, ICRA'04. 2004 IEEE International Conference on, IEEE, vol. 5, (2004), pp. 4393-4398.

[7] L. Doitsidis, K. P. Valavanis, N. C. Tsourveloudis and M. Kontitsis, "A framework for fuzzy logic based UAV navigation and control”, In Robotics and Automation, 2004. Proceedings, ICRA'04. 2004 IEEE International Conference on, IEEE, vol. 4, (2004), pp. 4041-4046.

[8] S. Kurnaz, O. Cetin and O. Kaynak, "Fuzzy logic based approach to design of flight control and navigation tasks for autonomous unmanned aerial vehicles", Journal of Intelligent and Robotic Systems, vol. 54, no. 1-3, (2009), pp. 229-244.

[9] M. Santos, V. Lopez and F. Morata, "Intelligent fuzzy controller of a quadrotor", In Intelligent Systems and Knowledge Engineering (ISKE), 2010 International Conference on, IEEE, (2010), pp. 141-146.

[10] K. Bickraj, T. Pamphile, A. Yenilmez, M. Li and I. N. Tansel, "Fuzzy Logic Based Integrated Controller for Unmanned Aerial Vehicles", In Florida Conference on Recent Advances in Robotics, FCRAR, (2006).

[11] G. M Hoffmann, H. Huang, S. L. Waslander and C. J. Tomlin, "Quadrotor helicopter flight dynamics and control: Theory and experiment", In Proc. of the AIAA Guidance, Navigation and Control Conference, vol. 2, (2007), p. 4.

[12] I. Sadeghzadeh and Y. M. Zhang, "A review on fault-tolerant control for unmanned aerial vehicles (UAVs)”, Infotech@ Aerospace, St. Louis, MO., (2011).

[13] Y. M. Zhang, A. Chamseddine, C. A. Rabbath, B. W. Gordon, C-Y. Su, S. Rakheja, C. Fulford, J. Apkarian, and P. Gosselin, "Development of advanced FDD and FTC techniques with application to an unmanned quadrotor helicopter testbed", Journal of the Franklin Institute, vol. 350, no. 9, (2013), pp. 2396-2422.

[14] V. Kumar and N. Michael, "Opportunities and challenges with autonomous micro aerial vehicles", The International Journal of Robotics Research, vol. 31, no. 11, (2012), pp. 1279-1291.

[15] Zemalache, K. Meguenni and H. Maaref, "Controlling a drone: Comparison between a based model method and a fuzzy inference system", Applied Soft Computing, vol. 9, no. 2, (2009), pp. 553-562. 
[16] O. Amidi, T. Kanade and R. Miller, "Vision-based autonomous helicopter research at Carnegie Mellon Robotics Institute 1991-1997”, American Helicopter Society, (1998)

[17] Y. Sun, Y. Sun, N. Xian, N. Xian, H. Duan and H. Duan, "Linear-quadratic regulator controller design for quadrotor based on pigeon-inspired optimization", Aircraft Engineering and Aerospace Technology, vol. 88, no. 6, (2016), pp. 761-770.

[18] I. Sadeghzadeh, A. Mehta, Y. Zhang and C.-A. Rabbath, "Fault-tolerant trajectory tracking control of a quadrotor helicopter using gain-scheduled PID and model reference adaptive control", In Annual conference of the prognostics and health management society, vol. 2, (2011).

[19] Ali, Z. Anwar, D. Wang and M. Aamir, "Fuzzy-Based Hybrid Control Algorithm for the Stabilization of a Tri-Rotor UAV", Sensors, vol. 16, no. 5, (2016), p. 652.

[20] Ali, Z. ain Anwar, D. Wang, M. Aamir and S. Masroor, "Trajectory Tracking of a Tri-Rotor Aerial Vehicle Using an MRAC-Based Robust Hybrid Control Algorithm”, Aerospace, vol. 4, no. 1, (2017), p. 3.

[21] A. Chamseddine, Y. Zhang, C.-A. Rabbath, C. Fulford and J. Apkarian, "Model reference adaptive fault tolerant control of a quadrotor UAV", AIAA Infotech@ Aerospace, St. Louis, Missouri, USA 2931, (2011).

[22] S. K. Hong, "Fuzzy logic based closed-loop strapdown attitude system for unmanned aerial vehicle (UAV)", Sensors and Actuators A: Physical, vol. 107, no. 2, (2003), pp. 109-118.

[23] M. Lasek, J. Pietrucha, M. Zlocka and K. Sibilski, "Analogies between rotary and flapping wings from control theory point of view”, In AIAA Atmospheric Flight Mechanics Conference and Exhibit, (2001), pp. 6-9.

[24] L. Hsu, "Variable structure model-reference adaptive control (VS-MRAC) using only input and output measurements: the general case", IEEE Transactions on Automatic Control, vol. 35, no. 11, (1990), pp. 1238-1243. 
International Journal of Control and Automation

Vol. 10, No. 10 (2017) 